\title{
Registerstudie zu lokalisierten GIST
}

Im Frühjahr 2010 wurde für Patienten mit gastrointestinalen Stromatumoren (GIST) die Registerstudie LOGIST gestartet. In einem Interview erläuterte Peter Reichardt, Bad Saarow, das Ziel dieser Registerstudie.

Herr Dr. Reichardt, was sind die Ziele dieser Registerstudie und warum ist es sinnvoll daran teilzunehmen?

Reichardt: Es gibt bereits eine internationale Registerstudie, GOLD reGISTry, die weltweit mehr als 1.000 Patienten mit fortgeschrittenem GIST sowie mehrere hundert Patienten mit lokalisiertem GIST eingeschlossen hat. Speziell für das lokalisierte Erkrankungsstadium wurde nun die deutsche, nicht-interventionelle Registerstudie LOGIST aufgelegt, in die alle Patienten mit lokalisiertem GIST eingebracht werden können, unabhängig davon, ob eine adjuvante Therapie durchgeführt wird oder nicht.

Ziel der Registerstudie ist es, den Status Quo der Behandlung lokalisierter GIST in Deutschland zu erheben.

- Welches Klassifikationssystem wird verwendet?
- Welche Patienten werden behandelt?

- Wer stellt die Indikation?

- Wer trifft die Therapie-Entscheidung?

- Wer führt die Behandlung durch?

- Welche Dosierung wird verwendet?

- Wie lange wird die Behandlung durchgeführt?

- Wiewerden die Kontrolluntersuchungen gemacht?

Über welchen Zeitraum ist die Registerstudie geplant und wie viele Patienten werden in die Studie eingeschlossen?

Reichardt: Es ist geplant, 400 Patienten im Laufe der nächsten zwei Jahre einzuschließen, die dann weitere zwei Jahre nachbeobachtet werden.

Quelle: Informationen der Novartis Pharma GmbH, Nürnberg, Interview mit: PD Dr. Peter Reichardt, Bad Saarow, Bericht: Dr. Susanne Heinzl, Reutlingen.

\section{Lungenkarzinom}

\section{Molekulardiagnostischer Test verfügbar}

Mit dem Epi proLung BL Reflex Assay ist seit dem 7. Juli 2010 in Europa ein neuartiger molekulardiagnostischer Test zur Unterstützung der Diagnose von Lungentumoren verfügbar. Der Einsatz des CE-gekennzeichneten Assays kann Pathologen und Klinikern helfen, eine bösartige Lungenerkrankung bei Patienten mit Verdacht auf ein Lungenkarzinom mit mehr Gewissheit zu bestätigen, wenn herrkömmliche diagnostische Verfahren z.B. keine schlüssigen Ergebnisse liefern.

Mit dem Epi pro Lung BL Reflex Assay wird methylierte DNS des SHOX2 (Short Stature Homeobox 2)-Gens in Bronchiallavage-Flüs- sigkeit nachgewiesen. Eine erhöhteDNS-Methylierung des SHOX2-Gens in der Bronchiallavage-Probe weist auf eine maligne Lungenerkrankung hin. Der klinische Nutzen des Tests wurde in einer Leistungsbewertungsstudie mit Probenmaterial von Patienten, die sich im Rahmen eines Forschungsprogramms an der Universität Liverpool/Großbritannien einer diagnostischen Abklärung des Verdachts auf ein Lungenkarzinom unterzogen hatten, gezeigt. In dieser Studie wurde bestätigt, dass methylierte SHOX2DNS ein sensitiver , spezifischer Biomarker für den Nachweis von Lungentumoren in Bronchiallavage-Flüssigkeit ist.

Quelle: Informationen der Epigenomics AG

\section{Bendamustin-Rituximab vielversprechend}

Die Kombination Bendamustin (Ribomustin ${ }^{\circledR}$, Mundipharma $\mathrm{GmbH}$ ) plus Rituximab (B-R) hat das Potenzial, ein neuer Standard für die Behandlung von Patienten mit nicht vorbehandelten follikulären, indolenten und Mantelzell-Lymphomen zu werden, sagte Mathias Rummel, Gießen, bei einer Joint Session der American Society of Clinical Oncology (ASCO) und der American Society of Hematology (ASH) anlässlich der ASCO-Jahrestagung 2010 in Chicago/USA. B-R war in einer PhaseIII-Studie der deutschen Studiengruppe indolente Lymphome (StiL) bei diesen Patienten deutlich wirksamer und besser verträglich als der bisherige First-line-Standard CHOP (Cyclophosphamid, Doxorubicin, Vincristin, Prednison)-R (Rummel MJ et al., 2009, ASH: \#405, Oral Presentation). Die StiL setzt B-R bereits als Standard in ihren neuen Studien ein, so Rummel. Auch andere Studiengruppen nutzen die vorliegenden Daten als Basis für ihre Studien: Die Nordic Lymphoma Group untersucht z.B. die Wirksamkeit und Sicherheit von B-R in Kombination mit Lenalidomid bei älteren Patienten mit Mantelzell-Lymphom (MCL4 [LENA-BERIT]-Studie).

\section{UV-Check-Dienst zur Hautkrebsprophylaxe}

Der Berufsverband der Deutschen Dermatologen (BVDD) hat in Zusammenarbeit mit dem Deutschen Zentrum für Luft- und Raumfahrt (DLR) und mit Unterstützung der Spirig Pharma AG einen UV-Check-Dienst entwickelt. Zu jedem zeitpunkt und für nahezu jeden Ort in Europa können hautbewusset User per Internet oder SMS die individuelle Eigenschutzzeit ihrer Haut in Echtzeit abrufen. Der Dienst steht unter www. uv-check.de kostenlos zur Verfügung. Zusammen mit vernünftigen Verhaltensmaßnahmen und hocwirksamen Sonnenschutzmitteln lassen sich somit Sonnenbrände vermeiden. 